\title{
MODELING AND CONTROL OF A MAGNETIC LEVITATION SYSTEM USING AN ANALOG CONTROLLER
}

\author{
Syed Mamun R Rasid*, Md. Belayet Hossain, Md. Emdadul Hoque, Md. Ariful Azam Arif and Md. Sarikat Ali Sarder \\ Department of Mechanical Engineering, Rajshahi University of Engineering \& Technology, Rajshai-6204, Bangladesh \\ *Corresponding authore-mail: mamun06me ruet@yahoo.com, smrasid.me@gmail.com
}

This is an open access article distributed under the Creative Commons Attribution License, which permits unrestricted use, distribution, and reproduction in any medium, provided the original work is properly cited.

\section{ARTICLE DETAILS}

\section{Article History:}

Received 29 July 2019

Accepted 30 August 2019

Available online 06 September 2019

\section{ABSTRACT}

This paper presents the modeling and control the position of the ferromagnetic ball in a controlled environment using analog controller where the current is varied in the electromagnet to keep the stable position of the ball. Generally, open loop magnetic levitation system is unstable non-linear system. Also, external disturbances are acting on the system making the control is very complex. First, analog controller based magnetic suspension system model is built to represent the dynamic behavior between the current of the electromagnetic coil and position of the ferromagnetic ball suspended. The model is developed using PID controller because it is the most popular industrial controller today. The PID controller is designed using pole assigned method so that pole of the designed system lies on the left half plane. The developed model is simulated using the proposed analog PID controller where step response and frequency response of the system is measured and made the system stable by tuning the proportional, integral and derivative parameter of the PID control. Then, experimental setup of the magnetic levitation system is developed and same control methodology is used to stable the position of the ferromagnetic ball as with simulation. Finally, the position of the levitated ball is suspended using the same control parameter of simulation.

\section{KEYWORDS}

Magnetic levitation system, PID control, Analog controller, Electromagnetic force.

\begin{abstract}
1. INTRODUCTION
Maglev is a technology to suspend the object in an air against gravity force by manipulating magnetic force without the support of mechanical system [1]. Magnetic suspension technology becoming increasingly popular because this system is contactless, low noise and low friction characteristics [2].Such systems are employed in the areas of high speed ground transportation systems, magnetic bearing [3], vibration isolation system [4], levitation of wind tunnels [5] etc.. Generally, magnetic suspension system is a typically non-linear and unstable in nature and are described by highly nonlinear differential equations. That's why, its modeling and control methodology attracts a lot of researchers around the world [2]. Many researchers have experimented the maglev technology using superconductor, permanent magnet and diamagnetic materials. But when the distance between the suspended object and magnet is too far, then the strength of magnetic field will not be enough to suspend the object. The approach is used to solve the problem by Electromagnet. The current passing through the coils of electromagnet produces varying magnetic field or force. Magnetic force produced from the electromagnet can attract any object in time domain [6].
\end{abstract}

In recent years, a considerable amount of study has been reported in the literature for controlling of maglev systems. All these studies are based on the controller design of the magnetic suspension of physical system's model. Different researcher have been used various control method to stable the magnetic suspension system. PID algorithms is most widely used controller in industry today because of its simplicity, ease of implementation and robustness [7]. [8] experimented a stabilized closed loop control of 1-DOF maglev using Proportional Integral Derivative (PID) and Linear Quadratic Regulator (LQR) controllers and examined the compare the stability performance of the maglev system under external disturbances. Again, Mundher H.A. Yaseen et al. [9] proposed a magnetic levitation system implemented under the effect of three types of controller which are Linear-quadratic regulator (LQR), proportional-integralderivative controller (PID) and Lead compensation and compared in term of three parameters Peak overshoot, Settling time and Rise time. Shekhar Yadav et al. [7] designed an optimized proportional-integral-derivative
(PID) controller using grey wolf optimizer (GWO) in frequency domain. Eng. Khalid Abdelhafiz Ali studied a magnetic levitation system [10] of iron sphere using analog PD controller. Apart from PID controller, several researchers have been used Fuzzy logic control with PID control or other controller alone. Shekhar Yadav [11] used Teaching Learning Based Optimization (TLBO) to optimize the parameter of the PID controller and compared the performance with the conventional control techniques. Tania Tariq Salim [12] presented a fuzzy logic controller design of linear magnetic levitation system and compared the performance of fuzzy logic control and Linear Quadratic Regulator Controller (LQRC) for the same system's model. Yemei Qina [2] built a state-dependent Autoregressive with exogenous input (SD-ARX) model. Also, State-dependent functional coefficients of the SD-ARX model are approximated by Gaussian radial basis function (RBF) neural networks.

In this study, an approach is presented to stable the ferromagnetic sphere ball using analog PID controller because of its popularity in industry although having some drawbacks. The cost of the digital controller is high so that analog PID controller will reduce the cost of the proposed control of magnetic levitation system. The objective of this study is to design and fabricate the magnetic levitation system, design the analog PID controller and stable the ball position by measuring the displacement of ball in time domain and frequency response of the ball under external disturbance.

\section{PRINCIPLES OF MAGNETIC LEVITATION SYSTEM}

Fig.1 shows the basic principles of the magnetic levitation system and Fig. 2 demonstrates the principle of magnetic levitation using block diagram. Maglev system consists of suspended object or plant, position sensor, actuator, power amplifier, controller etc. The system actuator consists of an electromagnet formed by wrapping an iron core of high permeability with an electrically conducting wire. The actuator creates a magnetic field when an electrical current is passed through the wires. This magnetic field creates an upward attractive force on any magnetic object placed below. The object to be suspended is placed between electromagnet and sensor placed below the ferromagnetic ball. A position sensor detects the position of the object and passes this information to the controller through 
reference. The controller then adjusts the current to the electromagnet actuator based on the object position to create a stable levitation of the suspended object.

\section{Electromagnet}

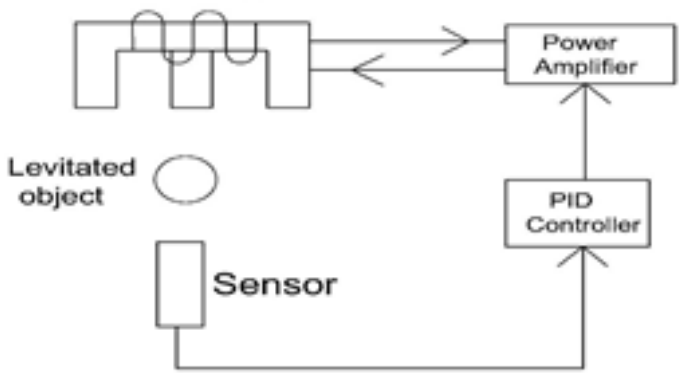

Figure 1: Basic principles of magnetic levitation system

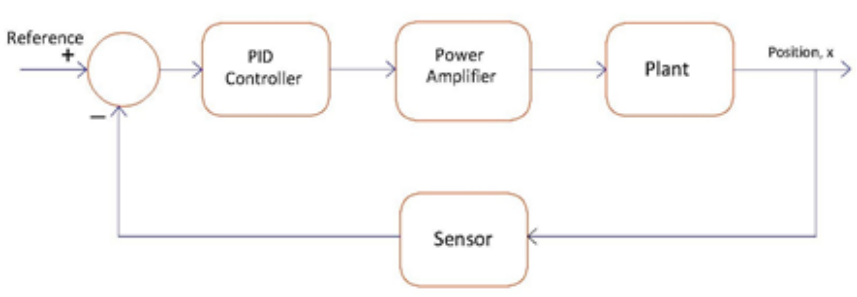

Figure 2: Closed loop block diagram of the magnetic levitation system

\section{CONTROLLER}

\subsection{PID Controller}

Magnetic Levitation system can be controlled by various ways. In this paper, PID controller is used to produce active feedback control system. PID controller is known as the proportional, integral and derivative controller. The popularity of PID is very high in control system society because it has special characteristics such as controlling capability [11]. It is commonly considered as an extreme form of a phase lead-lag compensator. The transfer function of the PID controller is given by

$U(s)=\left(k_{p}+k_{i} \frac{1}{s}+k_{d} s\right) E(s)$

where $\mathrm{kp}$ is the proportional gain, $\mathrm{ki}$ is the integral gain, $\mathrm{kd}$ is the derivative gain, $E$ is the error of the system and $U$ is the controller output. To find out the starting point of the PID controller, the parameters are tuned using ZN method due to its popularity and capability of providing the best result as compared with the other classical tuning criterion.

\subsection{Analog PID controller}

PID controller can be implemented by any of the followings, such as DSP, PLC, microcontroller and analog circuit. Here the controller is designed based on analog circuit with 'Op-Amp' as shown in Fig. 3. An analog controller is a device which implements the controller using analog signals to represents the loop parameters. The analog signal may be in the form of an electric current or a pneumatic air pressure. The front panel of an analog controller displays information for operators such as: measuring variables, error signals, output controller signal, and controller modes and parameters allows the adjustment of many parameters as the operator desires. The display is typically expressed in percentage of span (4-20 mA or 3-15 psi. Analog PID controller circuit consist of proportional, integrating and differentiating amplifier. The feedback from the sensor is inverted to boost up the voltage, when the object comes close to the sensor. The output is then passed through a proportional circuit to lift the object, integral circuit measure the error and a derivative circuit to dampen the vibration. After that the output from these three circuits is summed in another circuit and combined with an offset power supply. In this way, analog circuit works as PID controller circuit.

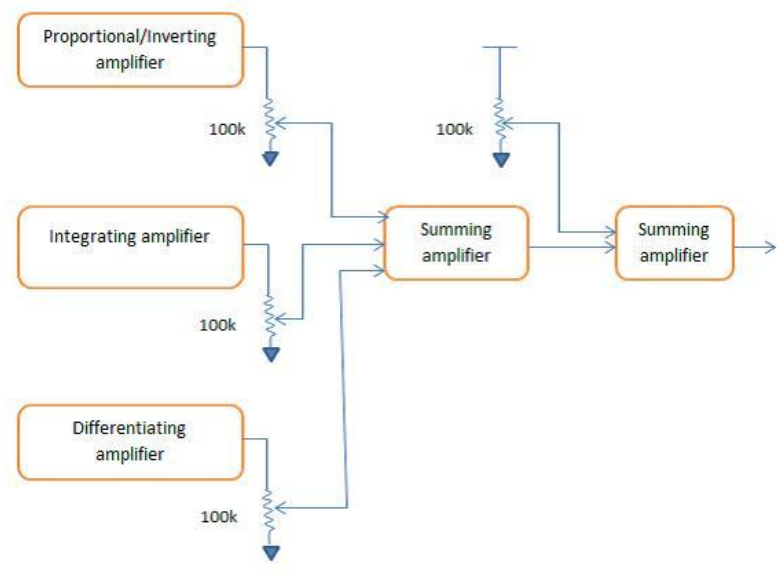

Figure 3: Block diagram of the analog PID controller

\section{MATHEMATICAL MODELING}

Using the fundamental principle of dynamics, the behavior of the ferromagnetic ball is given by the following electromechanical equation

$m \frac{d^{2} x(t)}{d t^{2}}=k_{a} i(t)+f_{d}$

where

$\mathrm{m}=$ mass of levitated object in $\mathrm{kg}$,

$\mathrm{x}(\mathrm{t})=$ displacement of the levitated object in $\mathrm{m}$,

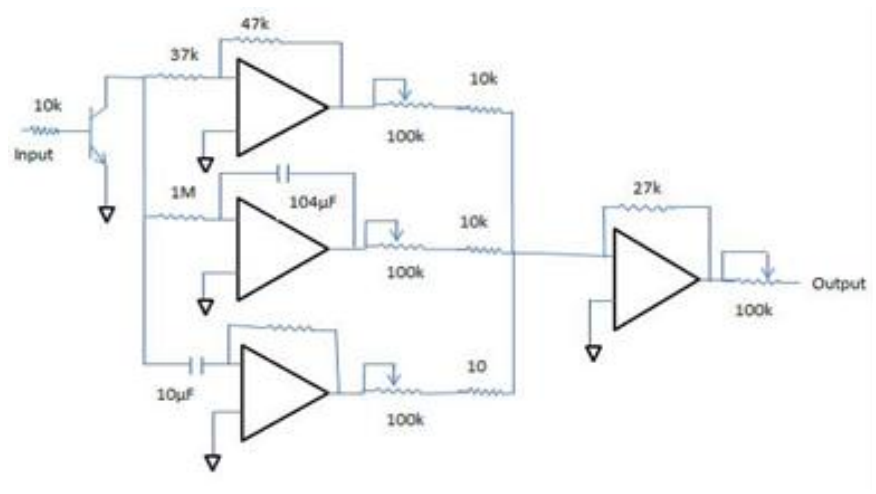

Figure 4: Circuit for analog PID controller

$\mathrm{ka}=$ force-current co-efficient of the electromagnet in N/A,

$\mathrm{i}(\mathrm{t})=$ current flowing through the electromagnet in Amp, $\mathrm{fd}=$ external disturbances on the levitated object in $\mathrm{N}$.

The open-loop transfer function of the MLS is oscillatory in nature i.e., either the metal ball attracted towards the electromagnetic coil or it may be fallen down. Therefore, there is a requirement of a controller which can effectively achieve the desired performances. An analog PID controller is used to achieve the desired performances.

The control current flowing through the electromagnet according to PID control is given by

$$
i(t)=\left\{k_{p} x(t)+k_{i} \int x(t)+k_{d} \frac{d}{d t} x(t)\right\}
$$

where, $\mathrm{kp}, \mathrm{ki}, \mathrm{kd}$ are the proportional, integral and derivative gain of the controller respectively.

Combining Eq. (i) and (ii) gives

$m \ddot{x}(t)+k_{a} k_{p} x(t)+k_{a} k_{i} \int x(t)+k_{a} k_{d} \frac{d}{d t} x(t)=f_{d}(t)$ 
Taking the Laplace transform of Eq. (iii) assuming all the initial conditions are zero can be written as

$$
\begin{aligned}
& m s^{2} x(s)+k_{a} k_{d} s x(s)+k_{a} k_{p} x(s)+k_{a} k_{i} \frac{1}{s} x(s)=f_{d}(s) \\
& \Rightarrow \frac{x(t)}{f_{d}(t)}=\frac{1}{m s^{2}+k_{a} k_{d} s+k_{a} k_{p}+k_{a} k_{i} \frac{1}{s}} \\
& =\frac{s}{m s^{3}+k_{a} k_{d} s^{2}+k_{a} k_{p} s+k_{a} k_{i}}
\end{aligned}
$$

From Eq. (iv), system characteristics equation is given by

$$
t_{c}(s)=m s^{3}+k_{a} k_{d} s^{2}+k_{a} k_{p} s+k_{a} k_{i}
$$

And the ideal characteristics equation of third order is given by

$$
\begin{aligned}
& t_{d}(s)=\left(s^{2}+2 \zeta \omega_{0}+\omega_{0}{ }^{2}\right)(s+\omega) \\
& \Rightarrow t_{d}(s)=s^{3}+s^{2} \omega_{0}(2 \zeta+1)+s \omega_{0}{ }^{2}(2 \zeta+1)+\omega_{0}{ }^{3}
\end{aligned}
$$

Co-relating the co-efficient of Eq. (v) and (vi), the gain value of the controller is given by

$$
\begin{aligned}
& k_{d}=\frac{\omega_{0}(2 \zeta+1)}{k_{a} m} \\
& k_{p}=\frac{\omega_{0}^{2}(2 \zeta+1)}{k_{a} m} \\
& k_{i}=\frac{\omega_{0}^{3}}{k_{a} m}
\end{aligned}
$$

This gain can be adjusted by changing the value of frequency and damping ratio of the designed system to stable the position of the levitated ball.

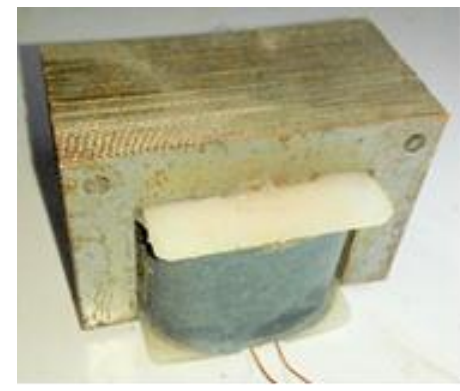

Figure 5: Electromagnet

\section{MAIN COMPONENTS}

The proposed magnetic levitation system consists of actuator, sensor, controller, power supply etc. The brief description and design specification of actuator and sensor given in the following.

\subsection{Actuator}

Electromagnet is used as actuator in the proposed levitation system. A type of magnet in which the magnetic field is produced by the flow of electric current is called electromagnet. The purpose of the magnetic actuator is to provide a force which will bear the load that is being supported. Since the bearing force is going to be magnetic, the object that is being suspended must be able to interact with magnetic forces. The advantage of an electromagnet is that the strength of the field is related to the amount of current flowing through the wire. Thus, a variable magnetic field is created to handle different loading conditions. The main consideration in the design of an electromagnet is its lifting power of the magnet. Cast steel was selected for this design because it has a narrow loop area which gives it a high permeability and fairly good coactivity, hence making it suitable for core of electromagnet [13].

Design specification of Electromagnet:

Shape of electromagnet: E-shape

Length of the core, Lc : $39 \mathrm{~mm}$

Width of the core, Dc : $26 \mathrm{~mm}$
Height of the core, $\mathrm{Hc}: 33 \mathrm{~mm}$ Wire specification, $20 \mathrm{AWG}$

Air gap: $5.84 \mathrm{~mm}$

Weight of the object, W: $0.113 \mathrm{~N}$

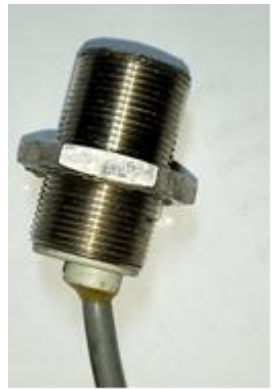

Figure 6: Inductive proximity sensor

\subsection{Sensor}

The electromagnet gives the user the ability to easily adjust the strength of the magnetic field, but one needs to know when and by how much to adjust the strength in a real application. The sensor provides this information. There are many different types of sensors that detect the distance between objects. Capacitive sensors, inductive sensors, optical sensors and acoustic sensors are just few of them. For the levitation of a steel ball a position sensor would be more suitable. The sensitivity of the sensor calibrated by making the setup and measured sensitivity is measured is $9.3 \mathrm{mv} / \mathrm{mm}$. Inductive proximity sensor is used in this system to measure position of levitated object which is shown in Fig. 6. When a metal object moves into the inductive proximity sensor's field of detection, Eddy circuits build up in the metallic object, magnetically push back, and finally reduce the Inductive sensor's own oscillation field. The sensor's detection circuit monitors the oscillator's strength and triggers an output from the output circuitry when the oscillator becomes reduced to a sufficient level.

\section{EXPERIMENTS}

\subsection{Experimental set-up}

A schematic diagram of the proposed magnetic levitation system is shown in Fig. 7. Also Fig. 8 shows the photograph of the experimental set-up used during experiment. The setup consists of a steel structure which has three stands. One rectangular and two circular plates are set one over another. The middle plate clamps the sensor and the upper plate holds the electromagnet. The height and diameter of the total setup is $30 \mathrm{~mm}$ and $15 \mathrm{~mm}$. Each stand is round shaped and of $8 \mathrm{~mm}$ diameter. Both the top and middle plates are fixed. The sensor is clamped such that its position can be varied by two identical hexagonal nuts when needed. A board containing controller circuits is attached to a box. The box also contains AC to DC converter circuits, power amplifier circuit, a DC to AC inverter. Steel is being used for its reliability.

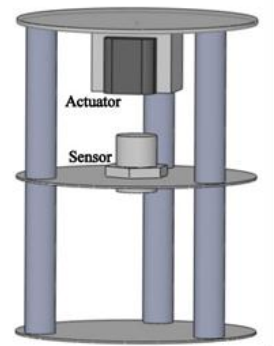

Figure 7: Schematic diagram of the proposed Maglev system

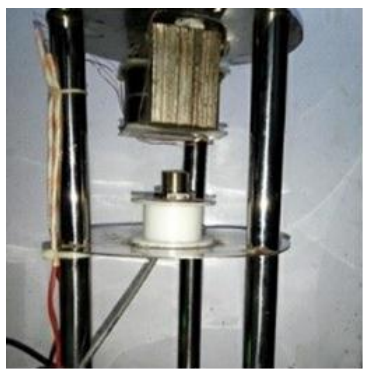

Figure 8: Photograph of the experimental apparatus 


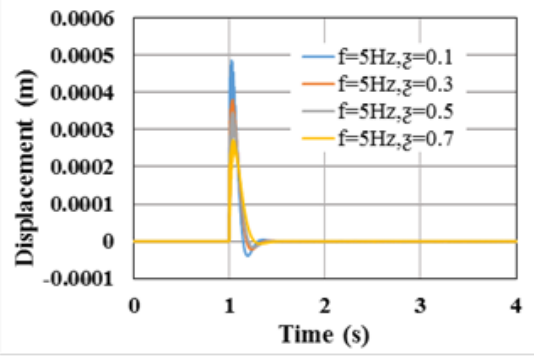

Figure 9: Step response curve for different designed parameter

\subsection{Results}

\subsubsection{Simulation results}

A simple Matlab Simulink model is developed to simulate the dynamic behavior of the system. The dynamic behavior of the model examined by measuring the step response of the system which is shown in Fig. 9 respectively. Step disturbance is applied on the system and displacement of the levitated is measured in time domain for different value of designed frequency and damping ratio of the closed loop system. Figure shows that when frequency and damping ratio is increased, the system becomes more stable because the pole of the closed loop magnetic levitation system is more on the left-hand side of the s plane.

\subsubsection{Experimental results}

It is seen from the Fig. 10 that the system is stable using analog PID controller. The levitation is shown in Fig.10 where the object is levitated at a stable position. The stable position of the ferromagnetic ball is achieved by using the same control parameter of the designed PID controller used in simulation. The position of the ball is also measured at the stable condition by taking the data from sensor. The data from the sensor is plotted is shown in Fig. 11. The displacement of the levitated ball is almost constant though there is small amount of oscillation. This vibration can be eliminated by several improvements to the system that could enhance the current configuration.

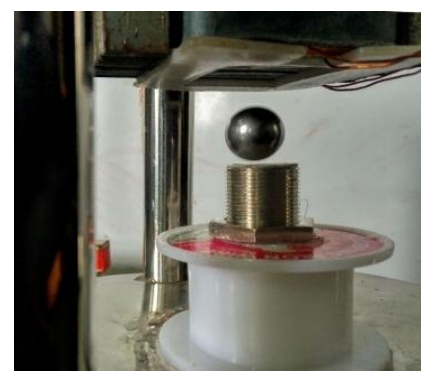

Figure 10: Maglev system with levitated ball

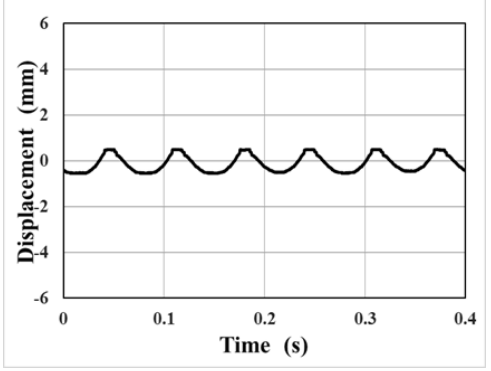

Figure 11: Displacement of the levitated ball in time domain

\section{CONCLUSION}

This paper investigates the modeling and control of magnetic levitation system using analog PID controller. The simplified mathematical model has been developed and transfer function has been established. The developed model is simulated using the designed PID control parameter and step response is measured which shows the stability of the system. Experiment is also conducted using the same control parameter and ferromagnetic ball is levitated in the stable position though there is small amount of oscillation. The stability of the ball is achieved using analog PID controller rather than digital PID controller which reduce the cost of magnetic levitation system.

\section{REFERENCES}

[1] Sahoo, N., Tripathy, A., Sharma, P. 2018. Single axis control of ball position in magnetic levitation system using fuzzy logic control. International Conference on Functional Materials and Chemical Engineering (Icfmce 2017), 323.

[2] Qin, Y.M. 2014. A modeling and control approach to magnetic levitation system based on state-dependent ARX model. Journal of Process Control, 24(1), 93-112.

[3] Valiente-Blanco, I. 2014. Characterization and Improvement of Axial and Radial Stiffness of Contactless Thrust Superconducting Magnetic Bearings. Tribology Letters, 54(3), 213-220.

[4] Chung, Y.D., Lee, C.Y., Park, E.Y. 2019. Design Considerations of Cooling Vessel to Improve Power Charging under Magnetic Shielding Structure in Wireless Power Charging System in Magnetic Levitation Train. Journal of Magnetics, 24(1), 112-117.

[5] Surva, S. 2015. Development of a simple MAGLEV system for a Lowspeed wind tunnel. Proceedings of the 2015 International Conference on Power and Advanced Control Engineering (Icpace), 441-444.

[6] Sun, Z. 2019. Nonlinear dynamic characteristics analysis of active magnetic bearing system based on cell mapping method with a case study. Mechanical Systems and Signal Processing, 117, 116-137.

[7] Yadav, S., Verma, S.K., Nagar, S.K. 2016. Optimized PID Controller for Magnetic Levitation System. Ifac Papersonline, 49(1), 778-782.

[8] Yaseen, M.H.A. 2017. A comparative study of stabilizing control of a planer electromagnetic levitation using PID and LQR controllers. Results in Physics, 7, 4379-4387.

[9] Yaseen, M.H.A., Abd, H.J. Modeling and control for a magnetic levitation system based on SIMLAB platform in real time. Results in Physics, 8, 153159.

[10] Ali, K.A., Abdelati, M. 2009. Magnetic Levitation of Iron Sphere Using Analog pd Controller. in AIP Conference Proceedings. 2009. AIP.

[11] Yadav, S., Verma, S.K. Nagar, S.K. 2018. Performance enhancement of magnetic levitation system using teaching learning based optimization. Alexandria Engineering Journal, 57(4), 2427-2433.

[12] Salim, T.T., Karsli, V.M. 2013. Control of Single Axis Magnetic Levitation System Using Fuzzy Logic Control. International Journal of Advanced Computer Science and Applications, 4(11), 83-88.

[13] Berry, M.V., Geim, A.K. 1997. Of flying frogs and levitrons. European Journal of Physics, 18(4), 307. 